\title{
Contract-based Time-of-use Pricing for Energy Storage Investment
}

\author{
Dongwei Zhao, Hao Wang, Jianwei Huang, Xiaojun Lin
}

\begin{abstract}
Time-of-use (ToU) pricing is widely used by the electricity utility. A carefully designed ToU pricing can incentivize end-users' energy storage deployment, which helps shave the system peak load and reduce the system social cost. However, the optimization of ToU pricing is highly non-trivial, and an improperly designed ToU pricing may lead to storage investments that are far from the social optimum. In this paper, we aim at designing the optimal ToU pricing, jointly considering the social cost of the utility and the storage investment decisions of users. Since the storage investment costs are users' private information, we design low-complexity contracts to elicit the necessary information and induce the proper behavior of users' storage investment. The proposed contracts only specify three contract items, which guides users of arbitrarily many different storage-cost types to invest in full, partial, or no storage capacity with respect to their peak demands. Our contracts can achieve the social optimum when the utility knows the aggregate demand of each storage-cost type (but not the individual user's type). When the utility only knows the distribution of each storagecost type's demand, our contracts can lead to a near-optimal solution. The gap with the social optimum is as small as $1.5 \%$ based on the simulations using realistic data. We also show that the proposed contracts can reduce the system social cost by over $30 \%$, compared with no storage investment benchmark.
\end{abstract}

\section{INTRODUCTION}

\section{A. Background and motivation}

Time-of-use (ToU) pricing is widely used by the electricity utility to shave the system peak load and reduce the system cost [1]. With ToU pricing, the utility divides one day into two or three fixed time periods. For example, in two-period ToU pricing, the utility sets a higher electricity price for the peak period (e.g., 4PM to 9PM) and a lower price for the off-peak period (e.g., 10PM to 3PM) [2].

The price difference between the peak and off-peak periods provides incentives for end-users' energy storage deployment, which can reduce their electricity bill [3]. Users with storage can purchase more electricity (by charging the storage) during the off-peak hours with a lower price. During the peak hours, users can discharge the storage to serve the demand with less electricity consumption from the utility at the higher price.

The increasing deployment of energy storage at end-user side, however, poses new challenges for the ToU pricing design. On one hand, a proper ToU pricing can incentivize users to invest in storage and reduce their energy cost, which

This work is supported by the Shenzhen Institute of Artificial Intelligence and Robotics for Society, and the Presidential Fund from the Chinese University of Hong Kong, Shenzhen.

Dongwei Zhao is with Department of Information Engineering, The Chinese University of Hong Kong. Hao Wang is with Department of Data Science and Artificial Intelligence, Monash University. Jianwei Huang is with the School of Science and Engineering, The Chinese University of Hong Kong, Shenzhen, and the Shenzhen Institute of Artificial Intelligence and Robotics for Society (AIRS) (corresponding author, e-mail: jianweihuang@cuhk.edu.cn). Xiaojun Lin is with School of Electrical and Computer Engineering, Purdue University. can further shave the system peak load and reduce the social cost (compared with no storage investment). On the other hand, if the ToU pricing design does not consider the storage impact and sets the price difference too high, it may incentivize too much storage investment and create a new and even higher system peak load. For example, if all the users invest in storage and shift the demand from the peak period to offpeak period, the original peak period will have zero demand while the original off-peak period will become the new peak. Furthermore, such an excessive storage investment may not be good for the social welfare, as some users may have high storage investment costs.

The above considerations motivate us to study the following problem: How should we design the ToU pricing to benefit users who invest in storage and achieve the social optimum that jointly considers the utility's supply cost and the users' storage investment costs? Notice that users can choose different storage products with different technologies, hence users can have heterogeneous storage costs. To reach the social optimum, we need to incentivize more users with low storage costs to invest in storage while discouraging users with high storage costs from investing. However, users' storage costs are often their private information, which poses challenges for the ToU pricing design. To solve this problem, we will use contract theory to elicit necessary information of users and induce the proper storage investment behavior.

\section{B. Related work}

There have been a substantial amount of works on designing ToU pricing for the utility. Chen et al. [4] offered optimal contract options of ToU pricing to households, which both minimizes the system peak load and maximizes the utility's profit. Kök et al. [5] studied optimal ToU pricing with the renewable energy investment. However, these works did not consider the possible impact of end-users' storage investment.

There are also works that studied the optimal storage operation and investment under ToU pricing. Nguyen et al. [6] optimized the operation of energy storage to minimize the users' cost under ToU pricing. Carpinelli et al. [7] proposed a probabilistic method to size the storage under ToU pricing. However, the ToU pricing is exogenously given in these works without considering the storage's impact on the system. So far, there is no literature studying the design of ToU pricing considering the end-users' storage investment.

In the ToU pricing design, we adopt contract theory to deal with users' private storage costs. Contract theory has been widely used in power systems as the mechanism for energy or service procurement. Most of the studies focused on the optimal contract for maximizing the payoff of the provider. 
For example, Tavafoghi et al. [8] designed an optimal contract of energy procurement for a strategic electricity seller. Haring et al. [9] proposed a contract that incentivizes users to offer demand response services. Some works also considered the contract for other objectives. Chen et al. [4] designed contracts for ToU pricing, which also considered minimizing the system peak load. However, the study in [4] focused on numerical solutions without theoretical optimality guarantee.

Different from existing studies, our study aims to analytically design effective yet simple contracts that minimize the social cost. The works [8] [9] designed a different contract item for each type of agents. However, in our work, we only need to design at most three common contract items for all types of users. This significantly reduces the complexity of the contract and makes it much more implementation friendly.

\section{Main results}

Our work focuses on the optimal contract design of the ToU pricing and end-users' storage investment, which aims to minimize the system social cost. The main contributions and results of this paper are listed as follows.

- Storage-aware ToU pricing: To the best of our knowledge, this is the first work to analytically study the socialoptimal ToU pricing design, considering the end-users' storage investment. The increasing storage deployment at the end-user side requires a new design of ToU pricing to maximize social welfare.

- Optimal contract design: It is challenging to optimize the ToU pricing with private user storage costs. We adopt contract theory to solve this problem, and only utilize the aggregate demand information of each type of storage cost. We analytically design two simple yet effective contracts TS-C and TS-I, under the complete and incomplete demand information of types, respectively. For an arbitrary number of types with diverse storage costs, we only need three contract items for each contract.

- Contract performance: We prove that Contract TS-C can achieve the social optimum when the utility knows the aggregate demand of each type. Based on realistic-data simulations, we show that even when the utility only knows the distribution (but not the exact value) of each type's demand, Contract TS-I can still lead to a near social optimum with a $1.5 \%$ gap with the social optimum.

- Benefits of contract: Via realistic-data simulation, we show that Contract TS-I can reduce the social cost by over $30 \%$, compared with a ToU pricing that provides low incentives and leads to no storage investment.

\section{System ModeL}

We consider one electric utility serving a group $\mathcal{I}=$ $\{1,2 \ldots I\}$ of users (e.g., residential users and business users). Figure 11 illustrates two timescales of decision making. At the beginning of an investment horizon of $D$ days (e.g., $D$ corresponding to many years), the utility announces the ToU pricing contract to users and users decide how much to invest in storage. The investment horizon is divided into operational horizons. Each operational horizon corresponds to one day, which is further divided into two periods $\mathcal{T}=\{p, o\}$ with peak

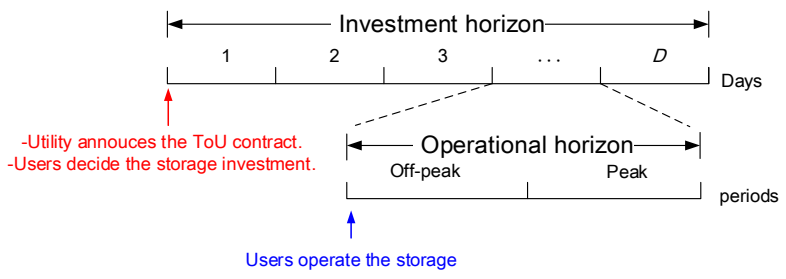

Fig. 1. Two timescales.

period $p$ and off-peak period $o$. On each day, each user utilizes storage to minimize his electricity cost. Next we will introduce the detailed models of the ToU pricing, users, and utility.

\section{A. ToU pricing}

We consider a two-period ToU pricing 11 commonly used by utilities [2], which is announced once and is valid for the entire investment horizon. For example, the peak period can be set from 4PM to 9PM and the off-peak period can be from 10PM to 3PM [2]. We assume that there are $H^{p}$ hours for the peak period and $H^{o}$ hours for the off-peak period, where $H^{p}+H^{o}=24$. The electricity prices of the peak and off-peak periods for user $i$ are $p_{i}^{p}$ and $p_{i}^{o}$, respectively. The peak price is no smaller than the off-peak price, i.e., $p_{i}^{p} \geq p_{i}^{o}$. Notice that we allow the utility to charge different users different prices.

\section{B. Users}

Users face the ToU pricing from the utility. Based on ToU pricing, users can invest (at the beginning of the investment horizon) and operate the storage (every day) to shift the demand. For each user $i$, we denote his total demand in the peak period (of a single day) as $D_{i}^{p}$ and the demand in the off-peak period (of a single day) as $D_{i}^{o}$. We assume that each user's demand pattern is the same across different days for analysis tractability ${ }^{2}$ Because of this, we do not need to use day index for the demands.

Next, we first explain the storage cost and electricity bill of users, and then we formulate and solve the user's cost minimization problem.

1) Storage cost: At the beginning of the investment horizon, each user $i$ decides the invested storage capacity $c_{i}$. The unit capacity cost of storage for user $i$ is $\theta_{i}$ per day, where we scale the total investment capacity cost of the entire investment horizon into one day ${ }^{3}$ Note that different users can choose different storage products with different technologies, hence users have heterogeneous private storage costs. User $i$ 's total storage cost per day is $\theta_{i} c_{i}$.

2) Electricity bill: Next, we calculate the daily electricity bill for users considering the storage deployment. During each day, in the off-peak period, if user $i$ purchase $s_{i}$ amount

${ }^{1}$ In practice, both two-period and three-period ToU pricing exist. We focus on the two-period pricing since it can already leverage the storage's impact, and we leave three-period case as future work.

${ }^{2}$ In the more realistic case, a user's demand will vary across days; such a general modeling will significantly complicate our analysis and hence will be left for future work.

${ }^{3}$ We can evenly divide the total capital cost by the number of days of the investment horizon considering zero annul interest rate. We also provide a scaling method with the annul interest rate in the online appendix [10]. 
of energy from the utility and charge the storage ${ }^{4}$ the total electricity consumption from the utility will be $D_{i}^{o}+s_{i}$. As a result, in the peak period, the total consumption from the utility will be $D_{i}^{p}-s_{i} \geq 05^{5}$ All the energy charged into the storage during the off-peak period will be discharged to serve demand in the peak period. Therefore, at the beginning of the off-peak period of the next day, the initial energy level of storage is zero. Then, user $i$ 's daily electricity bill is $p_{i}^{p}\left(D_{i}^{p}-s_{i}\right)+p_{i}^{o}\left(D_{i}^{o}+s_{i}\right)$. We consider $100 \%$ charge and discharge efficiency in our model, and we will generalize results to non-100\% efficiency in the future journal version.

3) User's cost minimization problem: Each user wants to minimize his total cost, which includes the electricity bill and the cost of storage investment (scaled into one day). If users are only charged based on the ToU pricing specified in Section II.A, then each user $i$ 's cost minimization problem is formulated in Problem UCM. Each user $i$ decides the invested storage capacity $c_{i}$ over the investment horizon and the charged energy $s_{i}$ in the off-peak period each day (where $s_{i}$ does not change over different days).

\section{Problem UCM: User $i$ 's Cost Minimization}

$$
\begin{aligned}
\min & p_{i}^{p}\left(D_{i}^{p}-s_{i}\right)+p_{i}^{o}\left(D_{i}^{o}+s_{i}\right)+\theta_{i} c_{i} \\
\text { s.t. } & 0 \leq s_{i} \leq c_{i} \\
& s_{i} \leq D_{i}^{p} \\
\operatorname{var}: & c_{i}, s_{i} .
\end{aligned}
$$

Constraint (1) ensures that the charge and discharge energy $s_{i}$ is within the storage capacity $c_{i}$. Constraint (2) prevents the negative demand of the user during the peak period.

We solve Problem UCM in Proposition 1. We let $p_{i}^{\Delta}$ denote the price difference between the peak price and off-peak price, i.e., $p_{i}^{\Delta} \triangleq p_{i}^{p}-p_{i}^{o}$.

Proposition 1 (Optimal solution to Problem UCM). The optimal solution to Problem UCM is as follows,

- If $\theta_{i}<p_{i}^{\Delta}, c_{i}^{*}=s_{i}^{*}=D_{i}^{p}$.

- If $\theta_{i}>p_{i}^{\Delta}, c_{i}^{*}=s_{i}^{*}=0$.

- If $\theta_{i}=p_{i}^{\Delta}, c_{i}^{*}$ can be any value in $\left[0, D_{i}^{p}\right]$, and $c_{i}^{*}=s_{i}^{*}$.

We will show the proofs of all mathematical results in the online appendix [10]. Proposition 1 shows that users' optimal storage investment decision has an all-or-nothing property. When the storage cost $\theta_{i}$ is smaller than the price difference $p_{i}^{\Delta}$, user $i$ will invest in the storage capacity equal to his peak demand $D_{i}^{p}$, which is also the charge and discharge energy of the storage. However, if the storage cost $\theta_{i}$ is higher than the price difference $p_{i}^{\Delta}$, no storage will be invested. Note that this optimal solution is derived under a simplified model of fixed demand across days. However, it can already capture the

${ }^{4}$ The payment in ToU pricing is based on the total consumed energy in peak and off-peak periods, without considering demand variation across hours. Thus, we only let the total charged and discharge energy be $s_{i}$. We assume users' charge and discharge of storage across hours within peak and off-peak periods can be regulated by the utility [11], so as to smooth the system load.

${ }^{5}$ We do not consider the negative demand in the current model, i.e., we do not allow users to sell back energy from the storage to the utility [11]. impact of ToU pricing on users' storage investment decisions ${ }^{6}$

\section{Electricity Utility}

The utility bears the energy supply cost of satisfying users' demand. We consider a quadratic supply cost, which is commonly used for thermal power plants [5]. Usually, the hourly energy, e.g., $\mathrm{kWh}$ or $\mathrm{MWh}$, is used to represent the power assuming the constant power within one hour. The supply cost for power $p_{t}$ in hour $t$ is given by $g\left(p_{t}\right)=\alpha p_{t}^{2}+\beta p_{t}+\gamma$, where the coefficients $\alpha>0, \beta>0$ and $\gamma>0$ are based on practical measurements, such as in [12]. Notice that the power consumption here are aggregated from all users.

Based on the quadratic supply cost, we approximate the power of the peak period and off-peak period (with multiple hours) by average energy per hour in these periods, respectively. For example, for the peak period of 12 hours with total demand $12 \mathrm{MWh}$, we use an average demand of $1 \mathrm{MW}$ per hour 7 Then, for the peak period, if the actual total demand is $L^{p}$ in the system, then the power of each hour is approximated by $L^{p} / H^{p}$. The total peak period's supply cost $g^{p}$ is then

$$
g^{p}\left(L^{p}\right)=\frac{\alpha}{H^{p}}\left(L^{p}\right)^{2}+\beta L^{p}+\gamma H^{p} .
$$

Similarly, the total supply cost for the demand $L^{o}$ in the offpeak period is

$$
g^{o}\left(L^{o}\right)=\frac{\alpha}{H^{o}}\left(L^{o}\right)^{2}+\beta L^{o}+\gamma H^{o} .
$$

\section{BENCHMARK: SOCIAL OPTIMUM}

We assume that the regulated utility aims at minimizing the system social cost [4], which includes users' storage costs and the utility's supply cost. In this section, we first study a benchmark where the utility as a social planner directly decides the optimal investment and operation of storage in the system, which will be later used in Section IV to compare with our contracts. In the benchmark, we will first describe different users types according to different storage costs. Then, we formulate and solve the social-cost minimization problem assuming the utility knows the aggregate demand of each type.

\section{A. Type model}

We assume a set of $\mathcal{K}=\{1,2 \ldots, K\}$ storage types with different costs in the market. The storage cost of type $k$ is denoted by $\theta^{k}$. Multiple users having the same storage cost belong to the same storage type. We rank the storage types with an increasing order of the storage costs, i.e., $\theta^{1}<\theta^{2}<$ $\cdots<\theta^{K}$. Note that the utility does not need to know an individual user's storage cost.

We assume that the utility can estimate the aggregate demand for each storage type, e.g., through surveys among users, historical data of storage incentive program [11], or market share of different storage products [14]. We denote the aggregate daily peak demand and off-peak demand for

${ }^{6}$ In reality, considering each user's varying demand across days, the threshold structure in Proposition 1 will still hold, although the threshold will depend on the demand distribution. We will generalize the results to the varying demand across day in the future journal version.

${ }^{7}$ Based the realistic load data of 40 users [13], the supply cost under average energy approximation has a small gap of less than $7 \%$ with the actual supply cost computed based on actual energy consumption per hour. This motivates us to consider the simplified average energy consumption model. 
storage type $k$ by $D^{k, p}$ and $D^{k, o}$, respectively. We consider the following two settings, depending on how much the utility knows the information of each type's demand.

- Complete demand information: The utility knows each storage type's aggregate demands at the beginning of the investment horizon.

- Incomplete demand information: The utility does not know the exact aggregate demands of each storage type, but knows the distributions of the demands, at the beginning of the investment horizon.

Next, we will consider two benchmarks of social cost minimization problem under complete demand information and complete demand distribution of types, respectively.

\section{B. Social cost minimization}

1) Complete demand information: We minimize the social cost in Problem SCM as below, where the utility as a social planner decides the optimal aggregate storage capacity $c^{k}$ and charge and discharge energy $s^{k}$ for all users of each type $k$.

The social cost includes the storage investment cost $\sum_{k \in \mathcal{K}} \theta^{k} c^{k}$ (scaled into one day), the supply cost $g^{p}$ in the peak period, and the supply cost $g^{o}$ in the off-peak period. The actual aggregate demand in the off-peak period is $\sum_{k \in \mathcal{K}}\left(D^{k, o}+s^{k}\right)$ due to the the charged energy $\sum_{k \in \mathcal{K}} s^{k}$ of all types, and the actual aggregate demand in the peak period is $\sum_{k \in \mathcal{K}}\left(D^{k, p}-s^{k}\right)$ with the discharged energy. The constraints follow the same structures as those in users' problem UCM, and we just replace the individual users with types.

Problem SCM: Social Cost Minimization under Complete Demand Information

$$
\begin{aligned}
& \min \sum_{k \in \mathcal{K}} \theta^{k} c^{k}+g^{p}\left(\sum_{k \in \mathcal{K}}\left(D^{k, p}-s^{k}\right)\right)+g^{o}\left(\sum_{k \in \mathcal{K}}\left(D^{k, o}+s^{k}\right)\right) \\
& \text { s.t. } 0 \leq s^{k} \leq c^{k}, \forall k \in \mathcal{K} \\
& \quad s^{k} \leq D^{k, p}, \forall k \in \mathcal{K} \\
& \text { var: } s^{k}, c^{k}, \forall k \in \mathcal{K} .
\end{aligned}
$$

Problem SCM is a quadratic programming problem. We will later characterize the structure of its optimal solution.

2) Incomplete demand information: For the incomplete demand information, we model the peak and off-peak demands of each type $k$ by random variables $D^{k}=\left(D^{k, p}, D^{k, o}\right)$. We focus on the joint distribution of all types' demand. We let $\boldsymbol{D}=\left(D^{1}, D^{2}, \ldots, D^{K}\right)$ in sample space $\mathcal{D}$ denote the joint random variable of peak and off-peak demand of all types. In the benchmark, we consider the ideal case that the social planner can decide the optimal storage investment and operation for each realization $\boldsymbol{D}$. This serves as a lower bound of the best performance for our contract. We formulate the expected social-cost minimization problem in Problem ESCM. Problem ESCM: Expected Social Cost Minimization under Incomplete Demand Information

$$
\begin{array}{r}
\operatorname{Sym}^{b}:=\min \mathbb{E}_{\boldsymbol{D}}\left[\sum_{k \in \mathcal{K}} \theta^{k} c^{k}(\boldsymbol{D})+g^{p}\left(\sum_{k \in \mathcal{K}}\left(D^{k, p}-s^{k}(\boldsymbol{D})\right)\right)\right. \\
\left.+g^{o}\left(\sum_{k \in \mathcal{K}}\left(D^{k, o}+s^{k}(\boldsymbol{D})\right)\right)\right]
\end{array}
$$
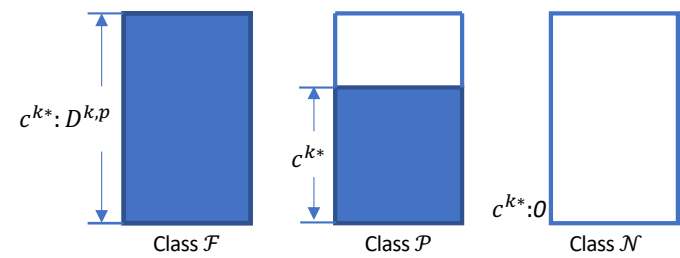

Fig. 2. Illustration of three classes.

$$
\begin{gathered}
\text { s.t. } 0 \leq s^{k}(\boldsymbol{D}) \leq c^{k}(\boldsymbol{D}), \forall k \in \mathcal{K}, \forall \boldsymbol{D} \in \mathcal{D}, \\
s^{k}(\boldsymbol{D}) \leq D^{k, p}, \forall k \in \mathcal{K}, \forall \boldsymbol{D} \in \mathcal{D}, \\
\text { var : } c^{k}(\boldsymbol{D}), s^{k}(\boldsymbol{D}), \forall k \in \mathcal{K}, \forall \boldsymbol{D} \in \mathcal{D} .
\end{gathered}
$$

Clearly, Problem ESCM can be decoupled into subproblems for each realization $\boldsymbol{D}$, which is equivalent to Problem SCM. The expected minimum costs of all these realizations will lead to the optimal objective value of Problem ESCM. Thus, we will just focus on Problem SCM and analyze it in next part.

\section{Solution structure of social cost minimization}

The optimal solution to Problem SCM divides storage types into three classes in terms of different users' storage investment behaviors. We show the three-class structure in Proposition 2, and present the detailed optimal solution to Problem SCM in the online appendix [10].

Proposition 2 (Three classes of storage types). The optimal solution of Problem SCM divides all storage types into three classes, which are denoted by sets $\mathcal{F}, \mathcal{P}$, and $\mathcal{N}$, respectively. The storage costs in Class $\mathcal{F}$ are smaller than the storage costs in Class $\mathcal{P}$, which are in turn smaller than those in Class $\mathcal{N}$.

- Class $\mathcal{F}$ : Users of each type $k \in \mathcal{F}$ fully invests in an aggregate storage capacity equal to the aggregate peak demand, i.e., $c^{k *}=D^{k, p}, \forall k \in \mathcal{F}$.

- Class $\mathcal{P}$ : There is at most one storage type in set $\mathcal{P}$, i.e., $|\mathcal{P}| \leq 1$. Users of this type partially invests in the aggregate storage capacity, i.e., it is strictly positive but strictly smaller than the peak demand, $0<c^{k *}<D^{k, p}, \forall k \in \mathcal{P}$.

- Class $\mathcal{N}$ : Users of each type $k \in \mathcal{N}$ invests in no storage, i.e., $c^{k *}=0, \forall k \in \mathcal{N}$.

Each of the class can be an empty set, with the constraint that $|\mathcal{F}|+|\mathcal{P}|+|\mathcal{N}|=|\mathcal{K}|$.

Proposition 2 classifies storage types into three classes in terms of different optimal storage investment decisions (that are centrally determined in the benchmark). Note that multiple users may belong to the same type. If user $i$ belongs to type $k$ and type $k$ is in any class $\mathcal{F}, \mathcal{P}$, or $\mathcal{N}$, we call that user $i$ is of class $F, P$, or $N$, respectively. Inspired by the three-class structure, we next design contracts to guide the three classes of users to invest in storage following the social-optimal solution.

\section{Contract Design}

We have introduced the benchmarks of social optimum, where the utility directly decides the aggregate storage investment for users of each type. In practice, however, users' storage costs are private information and they will not let the utility determine the storage investment on their behalf. Hence, we will design contracts for the utility to incentivize users. 
We will design two contracts, where Contract TS-C achieves the social optimum for the complete demand information of types, and Contract TS-I achieves near social optimum for the incomplete demand information of types

\section{A. Contract design: Complete demand information}

For the complete demand information of types, we will first introduce the contract and then characterize the conditions to ensure that the contract can reach social optimum.

1) Contract items: We will design Contract TS-C with three classes (i.e., items), and each item has two parameters: the peak and off-peak price difference of ToU pricing, and the constraint on each user's storage investment. The threeitem contract is motivated by the social optimum benchmark in Proposition 2 The two parameters are chosen according to the discussions in Proposition 1 of users' problem, where users' storage investment decision depends on the comparison between the price difference and the storage cost. Next we introduce the contract in detail.

Recall the utility knows the aggregate demand of each type under complete demand information. Then, based on the solution the benchmark Problem SCM, the utility knows the optimal aggregate storage investment of each type, and which type belongs to which class. However, the utility does not know which individual user belongs to which class. Therefore, we design the contract to incentivize users to reveal the class information. Specifically, for each user of class $x \in\{F, P, N\}$, we design the corresponding contract item $\left(p_{x}^{\Delta}, \eta_{x}\right)$. The parameter $p_{x}^{\Delta}$ is the price difference of ToU pricing ${ }^{8}$ The parameter $\eta_{x}$ is the maximum ratio of a user's invested storage capacity to his peak demand, computed as follows We let type $b$ be the only type in class $\mathcal{P}$, if $\mathcal{P}$ is non-empty.

- $\eta_{F}=1$ : Each user $i$ is required to invest in storage capacity no greater than his peak demand $D_{i}^{p}$.

- $\eta_{P}=c^{b *} / D^{b, p} \in(0,1)$ : Each user $i$ is required to invest in storage capacity no greater than $\eta_{P} D_{i}^{p}$.

- $\eta_{N}=0$ : Each user is not allowed to invests in storage.

We summarize the design of Contract TS-C as below. Next, we characterize the conditions of the price difference $p_{x}^{\Delta}, \forall x \in$ $\{F, P, N\}$ in Theorem 1 so as to ensure the social optimum.

2) Social-optima conditions: We show in Proposition 3 that the contract should satisfy two requirements to reach social optimum: (i) Each user only selects the contract intended for his class; (ii) Users corresponding to each contract item invest in storage capacity at the maximum ratio $\eta_{x}, x \in\{F, P, N\}$.

Proposition 3 (Social-optimum requirement). Contract $\boldsymbol{T S}$ - $\boldsymbol{C}$ achieves the social optimum if it induces users' behaviors that satisfy both Requirements (i) and (ii).

These two requirements will let the storage investment and operation of all users coincide with the social optimum. Next, we discuss how to achieve such two requirements in detail.

\footnotetext{
${ }^{8}$ Note that each contract item specifies only the price difference $p^{\Delta}$ but not the peak price $p_{i}^{p}$ and off-peak price $p_{i}^{o}$. We assume that the utility can set the peak and off-peak prices for each user $i$ in such a way that, if the user does not invest in storage, his payment is the same across all contract items, i.e., $p_{i, F}^{p} D_{i}^{p}+p_{i, F}^{o} D_{i}^{o}=p_{i, P}^{p} D_{i}^{p}+p_{i, P}^{o} D_{i}^{o}=p_{i, N}^{p} D_{i}^{p}+p_{i, N}^{o} D_{i}^{o}$, where $p_{i, x}^{p}\left(p_{i, x}^{o}\right)$ denotes the peak (off-peak) price for user $i$ in class $x$. As a result, only the price difference affects the user's selection of contract items.
}

Contract TS-C: Contract for ToU pricing and storage investment under complete demand information

1: Information: The utility knows the information of each type's aggregate demand.

2: Classification: The utility solves Problem SCM and divides storage types into three classes $\mathcal{F}, \mathcal{P}$ and $\mathcal{N}$, as stated in Proposition 2.

3: Contract items: The utility designs and announces threeitem contract: $\left(p_{x}^{\Delta}, \eta_{x}\right), \forall x \in\{F, P, N\}$ :

- $\eta_{F}=1, \eta_{P}=c^{b *} / D^{b, p}, \eta_{N}=0$.

- $p_{x}^{\Delta}, \forall x \in\{F, P, N\}$ satisfying Theorem 1

Requirement (i) is known as Incentive Compatibility (IC) in Definition 1. We denote the cost of user $i$ after choosing the contract item for class $x$ as $\pi_{i}\left(p_{x}^{\Delta}, \eta_{x}\right)$. Later we can show that our contract is always feasible even with such strict inequality.

Definition 1 (Incentive Compatibility). A contract is incentive compatible if user $i$ of class $x \in\{F, P, N\}$ minimizes his cost by choosing the contract intended for his class, i.e., for any $y \in\{F, P, N\}$ and $y \neq x, \pi_{i}\left(p_{x}^{\Delta}, \eta_{x}\right)<\pi_{i}\left(p_{y}^{\Delta}, \eta_{y}\right)$.

We choose the strict inequality in Definition 1 to ensure that a user always chooses the contract item intended for his class without any ambiguity. We can achieve Requirement (i) by satisfying Definition 1

We can achieve Requirement (ii) if we let the price difference be strictly larger than user $i$ 's storage $\operatorname{cost} \theta_{i}$ who is expected to invest in storage. This is implied by the all-ornothing property in Proposition 1

Then, in Theorem 1, we characterize the conditions on the price difference $p^{\Delta}$ such that Contract TS-C satisfies both Requirement (i) and (ii). Here, recall we use type $b$ to denote the only type in class $\mathcal{P}$ (if $\mathcal{P}$ is non-empty). We let Type $a$ be the type with the highest storage cost $\theta^{a}$ in class $\mathcal{F}$, and Type $c$ be the type with the lowest storage cost $\theta^{c}$ in class $\mathcal{N}$. If class $\mathcal{F}$ does not exist, we just set $p_{F}^{\Delta}=+\infty$ and $\theta^{a}=0$. If class $\mathcal{P}$ does not exist, we set $\eta_{P}=0$ and replace $\theta^{b}$ by $\theta^{c}$. If the class $\mathcal{N}$ does not exist, we set $\theta^{c}=+\infty$.

Theorem 1 (Social optima conditions). Contract TS-C achieves the social optimum, if and only if the price difference of each contract item satisfies all the following conditions:

- $p_{F}^{\Delta}: \eta_{P} \theta^{b}+\left(1-\eta_{P}\right) \theta^{a}<p_{F}^{\Delta}<\left(p_{P}^{\Delta}-\theta^{b}\right) \eta_{P}+\theta^{b}$.

- $p_{P}^{\Delta}: \theta^{b}<p_{P}^{\Delta}<\min \left(\theta^{c}, p_{F}^{\Delta} / \eta_{P}-\theta^{a}\left(1-\eta_{P}\right) / \eta_{P}\right)$.

- $p_{N}^{\Delta}: p_{N}^{\Delta} \geq 0$.

Furthermore, there always exist $p_{F}^{\Delta}, p_{P}^{\Delta}$, and $p_{N}^{\Delta}$ that satisfy the above conditions.

The conditions in Theorem 1 ensure that Requirement (i) and (ii) are satisfied. Theorem 1 suggests that the price difference cannot be too higher or too lower. Intuitively, a price difference that is too high (e.g., $p_{F}^{\Delta}>\left(p_{P}^{\Delta}-\theta^{b}\right) \eta_{P}+\theta^{b}$ ) may incentivize users to choose other classes' contract items (e.g., a class- $P$ user may choose the class- $F$ contract item), and a price difference that is too low cannot incentivize users to invest in enough storage. As long as the price difference 
$p_{x}^{\Delta}, \forall x \in\{F, P, N\}$ satisfies the conditions in Theorem 1. the contract will reach the social optimum. Due to the IC requirement, the conditions of $p_{F}^{\Delta}$ and $p_{P}^{\Delta}$ are coupled with each other. However, with the strict inequality of storage costs of types, i.e., $\theta^{a}<\theta^{b}<\theta^{c}$, conditions in Theorem 1 are always feasible.

\section{B. Contract design: Incomplete demand information}

In Section IV].A, we have designed a social-optimal Contract TS-C with complete information of each type's aggregate demand. When the utility only knows the distribution of such demands, we will also propose a three-item contract: Contract TS-I, which may not always achieve the social optimum of the benchmark Problem ESCM. We will evaluate the performance of the contract via the performance ratio with the benchmark.

1) Contract design: In Contract TS-I, we will still fix the three-class classifications across types. We first decide the optimal partition of the three classes $\mathcal{F}, \mathcal{P}$ and $\mathcal{N}$ by minimizing the expected social cost based on the demand distribution of types. Then, we design the contract following the same way as the case of complete demand information.

Specifically, recall that we let type $b$ be the only type in class $\mathcal{P}$. For each type $k \in\{1,2, \ldots, b-1\}$, we let the investment ratio $\eta^{k}=\eta_{F}=1$. For each type $k \in\{b+1, b+2, \ldots, K\}$, we let the investment ratio $\eta^{k}=\eta_{N}=0$. We denote the investment ratio of type $b$ by $\eta^{b}$. The utility decides the optimal type $b \in\{1,2, \ldots, K\}$ in class $\mathcal{P}$ and the optimal ratio $\eta^{b} \in$ $[0,1)$ to minimize the social cost as in the following Problem ESCM-C. The optimal ratio $\eta^{b}$ will be later set as $\eta_{P}$ in the contract. Note that we let $\eta^{b} \in[0,1]$ instead of $\eta^{b} \in(0,1)$ to ensure the feasibility of Problem ESCM-C. If $\eta^{b}=0$ or 1 , it will mean that there exists no class $\mathcal{P}$.

Problem ESCM-C: Expected Social Cost Minimization with Contract under Incomplete Demand Information

$$
\begin{array}{r}
\operatorname{Sym}^{c}:=\min \mathbb{E}_{\boldsymbol{D}}\left[\sum_{k=1}^{K} \theta^{k} \eta^{k} D^{k, p}+g^{p}\left(\sum_{k=1}^{K}\left(D^{k, p}-\eta^{k} D^{k, p}\right)\right)\right. \\
\left.+g^{o}\left(\sum_{k=1}^{K}\left(D^{k, o}+\eta^{k} D^{k, p}\right)\right)\right]
\end{array}
$$$$
\text { s.t. } \eta^{k}=1, \forall k \leq b-1 \text {, }
$$$$
\eta^{b} \in[0,1],
$$$$
\eta^{k}=0, \forall k \geq b+1,
$$$$
\text { var }: b \in \mathcal{K}, \eta^{b} \in[0,1] \text {. }
$$

Compared with the benchmark Problem ESCM, Problem ESCM-C fixes the three classes of types independently of demand's realization. Thus, there is a gap in the social cost between Problem ESCM-C and Problem ESCM.

Based on the solution to Problem ESCM-C, we design Contract TS-I following the way of Contract TS-C. The only difference between Contract TS-I and Contract TS-C is that the utility solves Problem SCM in designing Contract TS-C, while solving Problem ESCM-C in designing Contract TS-I. We show the detail of Contract TS-I in the online appendix [10]. Furthermore, we show in Theorem 2 that Contract TS-I achievs the optimal solution of Problem ESCM-C.
Theorem 2 (Optimality of Contract TS-I). Contract TS-I achieves the optimal solution of Problem ESCM-C.

Then, it only remains to solve Problem ESCM-C. Problem ESCM-C is a mixed integer quadratic programming. We solve it by an exhaustive search over the boundary types, which is efficient since we only compare $K$ results. We show the detailed algorithm in the online appendix [10].

2) Performance metric: We evaluate Contract TS-I performance based on the ratio $\kappa$, which is the ratio between the social cost induced by Contract TS-I and the minimum social cost of the benchmark Problem ESCM, i.e.,

$$
\kappa=\frac{\text { Sym }^{c}}{\text { Sym }^{b}} \text {. }
$$

Recall that $S y m^{b}$ is a lower bound of the social optimum under incomplete demand information of types and thus $\kappa \geq 1$. When $\kappa$ is closer to 1 , Contract TS-I performs closer to the social optimum. In the next section, we will use the real data to construct the demand distribution, and we show that the ratio $\kappa$ can be very close to 1 in practice.

\section{Simulation Result}

We have shown that for types' complete demand information, Contract TS-C achieves the social optimum. For the incomplete demand information, we will further show Contract TS-I's performance by constructing the type's demand distribution using the realistic data of users' load and solar energy. We show that Contract TS-I can lead to the social cost that is very close to the benchmark Problem ESCM.

\section{A. Setup}

1) Load profile: Based on the Austin Data set [13], we pick hourly load and solar energy generations of 40 (households) users in one year (with valid data of 361 days). In Figure 3 (a), we show the aggregate energy profile with seven randomly picked days in one year, where the blue curves and red curves represent the aggregate loads and solar energy generations, respectively. In Figure 3 (b), we show the aggregate net load (load minus solar energy) $)^{9}$ of seven randomly picked days in blue curves, with the mean value computed based on the entire year's data in green curve. We construct the users demand distribution based on their net loads profiles of the entire year.

2) Peak and off-peak periods of ToU pricing: Based on the average net load of all users in Figure 3 (b), we empirically set the peak period from 18:00 to 00:00 (7 hours), and the off-peak period from 01:00 to 17:00 (17 hours).

3) Storage cost: We consider 4 storage types with the corresponding (daily) investment costs of $\left[\theta_{1}, \theta_{2}, \theta_{3}, \theta_{4}\right]=[\bar{\theta}(1-$ $\left.\left.1.5 \lambda^{s}\right), \bar{\theta}\left(1-0.5 \lambda^{s}\right), \bar{\theta}\left(1+0.5 \lambda^{s}\right), \bar{\theta}\left(1+1.5 \lambda^{s}\right)\right]$. The mean value of the storage costs is $\bar{\theta}$. The coefficient $\lambda^{s}$ indicates the diversity of storage costs among types ${ }^{10}$ We scale the storage cost for years evenly into one day, ranging from $2 \$ / \mathrm{MWh}$ (for CAES) to $130 \$ / \mathrm{MW}$ (for Tesla Powerwall). In simulations,

\footnotetext{
${ }^{9}$ We let users curtail the surplus renewable energy in simulations.

${ }^{10}$ Storage costs can be very diverse. According to $[15]$, the compressed-air energy storage (CAES) has the cheap capital cost about $53-84 \$ / \mathrm{kWh}$ with the lifespan of 20-100 years. The Lithium battery's cost can be high. Typically, Tesla Powerwall's price is $6500 \$$ for $13.5 \mathrm{kWh}$ with the warranty of 10 years.
} 


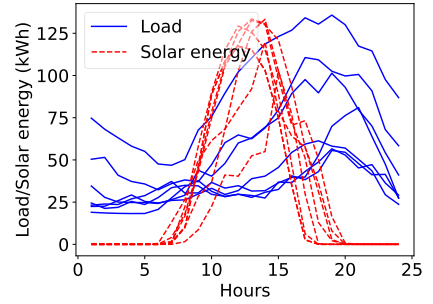

(a)

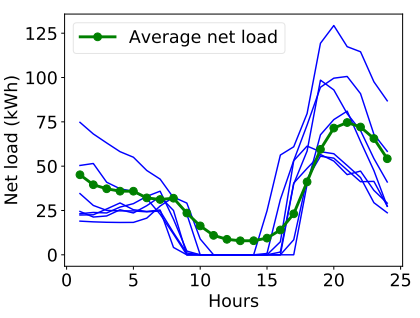

(b)
Fig. 3. (a) Aggregate load/solar energy; (b) Aggregate net load.

we randomly group 40 users into 4 types, and calculate the mean of 1000 such random groupings of storage types.

\section{B. Performance of the proposed contract}

Based on the empirical net-load data, we will show that Contract TS-I leads to social cost that is very close to the benchmark Problem ESCM, and such a contract significantly reduces the social cost compared with no storage investment.

Figure 4(a) shows the average ratio $\kappa$ of all simulated random type groupings with different average storage costs $\bar{\theta}$. Different curves correspond to different penetration levels of solar energy generations, where level 0 means no solar energy, level 1 means the solar energy in the data set, and level 2 doubles the solar energy in the data set (which represents a future scenario with high renewable penetration). The shaded regions correspond to the one-standard-deviation ranges of all simulated type groupings. Based on Figures 4(a), we have the following observations. First, Contract TS-I leads to a social cost that is very close to the benchmark Problem ESCM, and the average ratio $\kappa$ is always below 1.015. Second, Contract TS-I is robust among different type groupings. The standard deviation of the ratio $\kappa$ in the simulated random type groupings is smaller than 0.005 .

We define $\kappa^{n o}$ as the ratio between the social costs of no storage investment and Contract TS-I. The reason for considering the no storage investment case is that current ToU prices in many places (e.g., most states in US [16]) are not high enough to incentivize storage investment. Figure 4(b) shows the average ratio $\kappa^{n o}$ with different average storage costs $\bar{\theta}$. Different curves correspond to different penetration levels of solar energy. The shaded regions correspond to the one-standard-deviation ranges of all simulated random type groupings. Compared with the ToU pricing that leads to no storage investment in the system, Figure 4(b) shows that Contract TS-I can significantly reduce the social cost, especially when the renewable energy penetration level is high and the storage cost is low. For example, when the solar energy amount is doubled and the average storage cost is below $15 \$ / \mathrm{MWh}$, the social cost can be reduced by over $30 \%$.

\section{CONCLUSION}

In this paper, we design two contracts of the ToU pricing considering the storage investment, which deals with the issue of users' private storage costs. Both contracts contain only three contract items, which guides users of different storage costs to invest in full, partial, or no storage capacity . We show that Contract TS-C can achieve the social optimum under

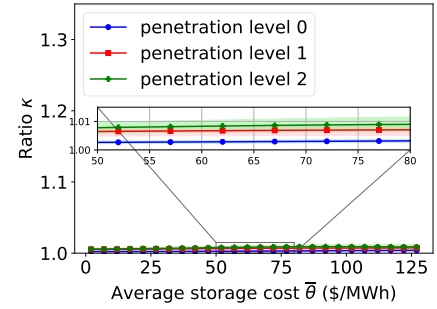

(a)

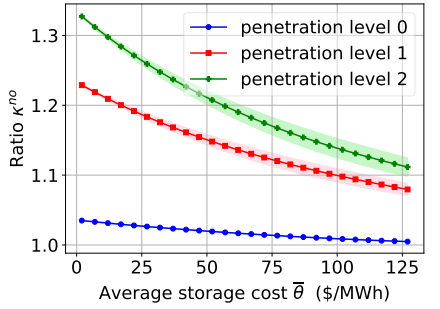

(b)
Fig. 4. Contract TS-I performance: (a) Ratio $\kappa$; (b) Ratio $\kappa^{n o}$. Both are with average storage cost $\bar{\theta}$ at $\lambda^{s}=1 / 3$.

complete information of the aggregate demand of each type. Via simulations based on realistic data, we show that Contract TS-I can achieve near social optimum when the utility only knows the demand distribution of each type.

For the future work, we plan to consider the more general case where users' demands can vary across days. We expect some of our main conclusions can still hold. For example, users will still be classified into several classes in terms of the invested storage capacity as a function of their demands, based on which contracts can be designed.

\section{REFERENCES}

[1] Y. Hung and G. Michailidis, "Modeling and optimization of time-of-use electricity pricing systems," IEEE Trans.on Smart Grid, vol. 10, no. 4, pp. 4116-4127, 2019.

[2] "Time of use plans,", acessed on 2020.5.20, https://www.sce.com/residential/rates.

[3] A. J. Pimm, T. T. Cockerill, and P. G. Taylor, "Time-of-use and timeof-export tariffs for home batteries: Effects on low voltage distribution networks," Journal of Energy Storage, vol. 18, pp. 447-458, 2018.

[4] S. Chen, H. Alan Love, and C. Liu, "Optimal opt-in residential timeof-use contract based on principal-agent theory," IEEE Transactions on Power Systems, vol. 31, no. 6, pp. 4415-4426, 2016.

[5] A. G. Kök, K. Shang, and Ş. Yücel, "Impact of electricity pricing policies on renewable energy investments and carbon emissions," Management Science, vol. 64, no. 1, pp. 131-148, 2018.

[6] T. A. Nguyen and R. H. Byrne, "Maximizing the cost-savings for time-of-use and net-metering customers using behind-the-meter energy storage systems," in 2017 NAPS, 2017, pp. 1-6.

[7] G. Carpinelli, F. Mottola, and D. Proto, "Probabilistic sizing of battery energy storage when time-of-use pricing is applied," Electric Power Systems Research, vol. 141, pp. 73-83, 2016.

[8] H. Tavafoghi and D. Teneketzis, "Optimal contract design for energy procurement," in 2014 52nd Annual Allerton Conference on Communication, Control, and Computing (Allerton), Sep. 2014, pp. 62-69.

[9] T. Haring and G. Andersson, "Contract design for demand response," in IEEE PES Innovative Smart Grid Technologies, Europe, 2014, pp. 1-6.

[10] "Appendix: Joint contract design for time-of-use pricing and energy storage investment." [Online]. Available: https://shorturl.at/jrFH2

[11] "Discover the self-generation incentive program, accessed 2019.12.2, https://www.selfgenca.com/home/resources/."

[12] L. Wu, "A tighter piecewise linear approximation of quadratic cost curves for unit commitment problems," IEEE Transactions on Power Systems, vol. 26, no. 4, pp. 2581-2583, Nov 2011.

[13] "Dataport from pecan street," acessed on 2020.5.20, https://dataport.cloud.

[14] "Energy storage market - growth, trends, and forecast (2020 - 2025), accessed 2019.12.8, https://www.mordorintelligence.com/industryreports/energy-storage-market."

[15] P. Ralon, M. Taylor, A. Ilas, H. Diaz-Bone, and K. Kairies, "Electricity storage and renewables: Costs and markets to 2030," International Renewable Energy Agency: Abu Dhabi, United Arab Emirates, 2017.

[16] J. Finkelstein, S. Kane, and M. Rogers, "How residential energy storage could help support the power grid," McKinsey and Company, 2019. 